\title{
Síndrome compartimental no primeiro interósseo dorsal: relato de caso
}

\author{
Compartment syndrome in the first dorsal interosseous compartment: case report \\ Clara Wilma Fernandes Rosendo ${ }^{1}$ (D) , José Rodolfo Lopes de Paiva Cavalcanti² (D), \\ Raimundo Rosendo de Oliveira ${ }^{3}$ (D)
}

\begin{abstract}
Resumo
O diagnóstico da síndrome compartimental em regiões que não são frequentemente afetadas, por possuírem uma boa relação continente-conteúdo, pode se tornar difícil, uma vez que o cirurgião terá dificuldades em alcançar um diagnóstico apenas por um sinal ou sintoma isolado. Assim, muitas vezes, pode-se protelar a conduta adequada, desencadeando danos ao paciente. A paciente era uma mulher, de 29 anos, que foi atendida com dor em mão esquerda por queimadura, com lesão em região anterior à tabaqueira anatômica de tamanho significativo. A paciente já havia sido submetida a cirurgia na sua cidade de origem com desbridamento de pele e tecido subcutâneo há 30 dias, com evolução sem melhora do quadro doloroso. Estava em uso de antibioticoterapia (ceftriaxona 1 g de 12 em 12 horas) e analgesia com dose terapêutica de morfina de 8 em 8 horas.
\end{abstract}

Palavras-chave: síndromes de compartimento; fasciotomia; queimaduras.

\begin{abstract}
In areas that are not commonly affected by compartment syndrome because they have a good content/container ratio, diagnosis of the condition can be a challenge, since surgeons will find it difficult to make a diagnosis on the basis of an isolated sign or symptom. As a result, the correct treatment can very often be delayed, causing harm to the patient. In this case, the patient was a 29-year-old woman who was seen for a painful left hand secondary to a large burn injury to the area anterior of the anatomical snuffbox. She had already undergone surgery in her home town 30 days previously, with debridement of skin and subcutaneous tissue, but the pain had not improved. She was on antibiotic therapy (ceftriaxone, $1 \mathrm{~g}$ every 12 hours) and analgesia, with therapeutic morphine doses every 8 hours.
\end{abstract}

Keywords: compartment syndromes; fasciotomy; burns.

Como citar: Rosendo CWF, Cavalcanti JRLP, Oliveira RR. Síndrome compartimental no primeiro interósseo dorsal: relato de caso. J Vasc Bras. 2021;20:e20200094. https://doi.org/10.1590/1677-5449.200094

\footnotetext{
${ }^{1}$ Universidade Federal do Rio Grande do Norte - UFRN, Departamento de Ciências da Saúde, Natal, RN, Brasil.

${ }^{2}$ Universidade do Estado do Rio Grande do Norte - UERN, Mossoró, RN, Brasil.

${ }^{3}$ Hospital Regional Tarcísio Maia - HRTM, Departamento de Cirurgia Geral e Vascular, Mossoró, RN, Brasil.

Fonte de financiamento: Nenhuma.

Conflito de interesse: Os autores declararam não haver conflitos de interesse que precisam ser informados.

Submetido em: Junho 02, 2020. Aceito em: Outubro 05, 2020.
}

O estudo foi realizado no Hospital Regional Tarcísio Maia (HRTM), Mossoró, RN, Brasil. 


\section{INTRODUÇÃO}

As referências acerca da síndrome do compartimento tratam do envolvimento de estruturas musculares e nervosas fragilizadas por disfunções motoras e sensoriais. Esse quadro patológico refere-se a uma desproporção entre continente e conteúdo, devido à qual essas estruturas sofrem injúria, levando a sequelas irreversíveis de acordo com o grau de comprometimento caso não sejam tratadas adequadamente e em tempo hábil.

Os cirurgiões vasculares estão acostumados a vivenciar essa patologia principalmente em membros superiores e inferiores. Porém, qualquer região do corpo é passível de ser acometida por essa síndrome. O diagnóstico resulta de uma avaliação clínica cuidadosa e normalmente dispensa métodos diagnósticos especializados, com o intuito predominante de evitar graves complicações, a exemplo da inviabilidade motora.

\section{DESCRIÇÃO DO CASO}

Esse estudo atendeu a todas as recomendações preconizadas pela resolução 466/2012 - CNE. Foi devidamente avaliado e aprovado pelo Comitê de Ética em Pesquisa (CAAE 31017920.7.0000.5294 e protocolo 4.050.507).

Uma paciente do sexo feminino, de 29 anos, deu entrada em um pronto-socorro queixando-se de dor intensa na mão esquerda por queimadura com café quente. No momento em uso de morfina para alívio do sintoma, apresentou ao exame físico lesão ao nível da região anterior à tabaqueira anatômica de tamanho significativo com crosta áspera cobrindo toda a lesão, associada a sinais de edema na mão e impotência funcional dos quirodáctilos, além de reação de dor de forte intensidade à manipulação.

Há quatro semanas, a paciente já havia sido submetida à desbridamento cirúrgico na sua cidade de origem, porém não apresentou melhora do quadro doloroso e estava em uso contínuo de morfina, sem resultados satisfatórios. Não havia sinais de infecção local.

Em nosso serviço, realizamos novo desbridamento da lesão, a qual apresentou-se sangrante, com sinais iniciais de granulação e alívio das dores, recebendo alta hospitalar em seguida (Figura 1).

Após três dias, houve retorno ao consultório por agravamento da dor e foi observada nova crosta necrótica cobrindo a ferida. Procedeu-se com internamento da paciente, para imediata conduta cirúrgica sob anestesia de plexo braquial, exploração profunda da ferida e abertura da fáscia dorsal da mão para exposição dos músculos interósseos dorsais, nos quais foi observado aspecto pálido e pontos de necrose que herniavam ao progredir com a abertura da fáscia, havendo necessidade de ressecção da musculatura necrótica. A lesão foi ampliada em todos os sentidos para exploração de outros músculos em busca de algum comprometimento (Figura 2).

No pós-operatório da paciente, houve evolução sem dores, sem uso de medicamentos analgésic os e com apresentação de sinais de granulação na ferida. Ela recebeu alta hospitalar depois de 48 horas. Após

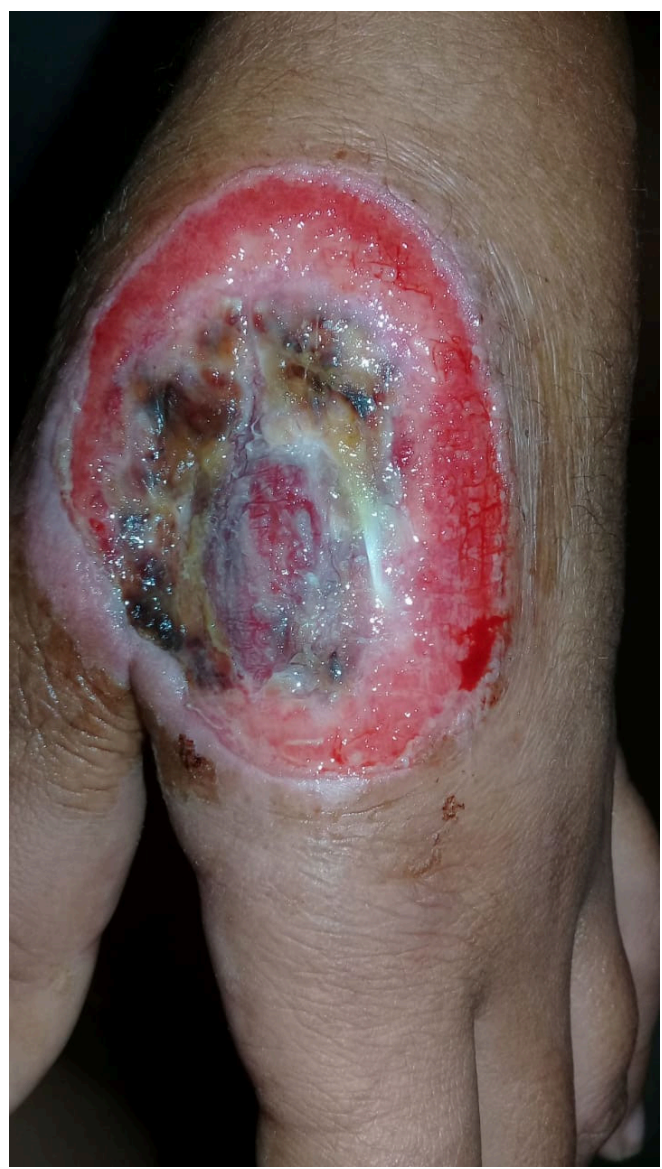

Figura 1. Imagem pós-operatória mostrando lesão sangrante e com sinais de granulação.

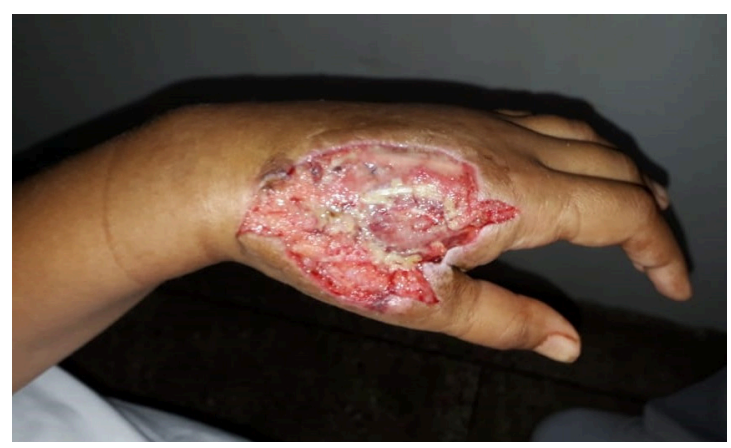

Figura 2. Exposição dos músculos interósseos dorsais, com pontos de necrose. 


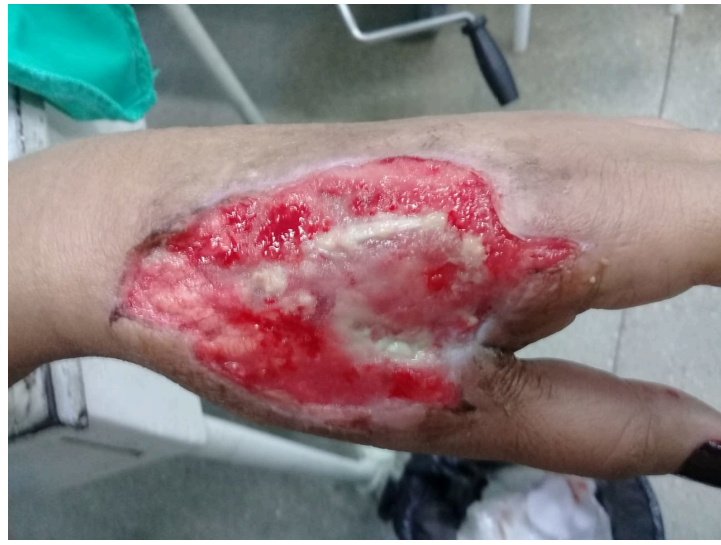

Figura 3. Paciente relatou melhora dos sintomas álgicos, e a medicação analgésica foi suspensa.

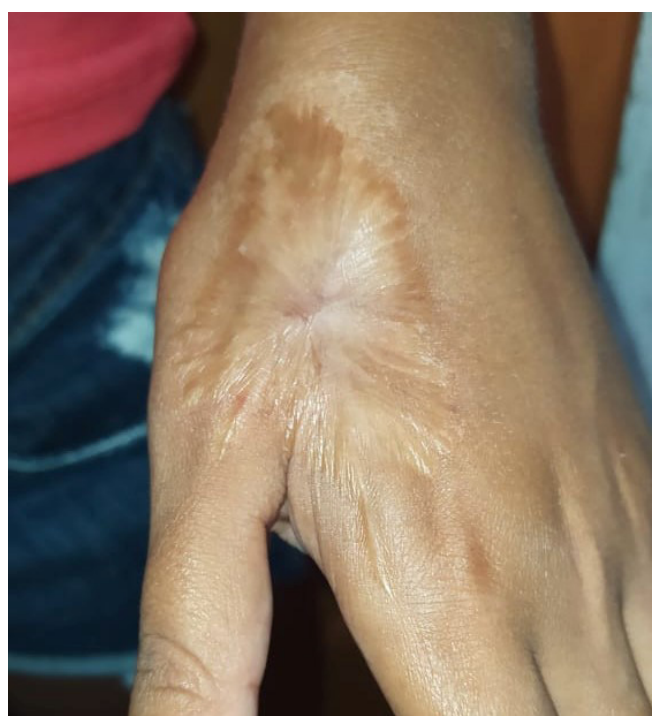

Figura 4. Ferida cicatrizada na região anterior à tabaqueira anatômica com tecido cicatricial fino mostrando enrugamento dos bordos, porém totalmente cicatrizada.

uma semana, a paciente retornou para o consultório, sendo encaminhada para fisioterapia motora e para procedimento de cirurgia plástica para preenchimento de enxerto dermoepidérmico (Figura 3). A Figura 4 mostra a lesão cicatrizada após os procedimentos cirúrgicos.

\section{DISCUSSÃO}

O mecanismo fisiopatológico da síndrome do compartimento em queimaduras de segundo grau profundas ou de terceiro grau envolvendo extremidades, como mãos e pés, pontua um aumento da pressão tissular nos compartimentos musculares associado aos sinais clássicos de inflamação de rubor e edema ${ }^{1}$. Como resultado, a elevação pressórica observada comprime estruturas vasculares e nervosas, gerando sinais - restrição ao movimento, cianose e hipoperfusão - e sintomas - dor intensa e parestesia - condizentes com o quadro resultante de redução da irrigação sanguínea do membro e imobilidade. Um quadro álgico desproporcional e resistente ao tratamento analgésico convencional associado ao edema pronunciado constitui a base para o estabelecimento do diagnóstico clínico da síndrome compartimental, apesar de serem sintomas genéricos presentes em inúmeros contextos de trauma ${ }^{2}$.

Um estudo apontou que a obtenção de $90 \%$ de liberação fascial pode acarretar um efeito desejado na diminuição da pressão intracompartimental a ponto de atingir um valor próximo ao basal ${ }^{3}$. Assim, mediante a descompressão do compartimento muscular comprometido via fasciotomia, o propósito do cirurgião vascular deve ser restabelecer a circulação funcional, visto que, depois de determinado período, a realização desse procedimento frequentemente torna-se ineficaz para a obtenção de uma melhora clínica considerável, podendo surgir como sequela a perda funcional ou anatômica do membro afetado pela síndrome ${ }^{4}$. Logo, pontua-se de extrema importância o diagnóstico precoce da síndrome compartimental em um paciente vítima de queimadura e outras situações possivelmente agravantes, além da implementação de medidas para o alívio da situação do paciente, como o desbridamento de tecidos necrosados, a drenagem de abscessos e a fasciotomia.

Essa síndrome apresenta uma incidência de 3,1 a cada 100.000 habitantes. Entre as suas causas, caracteriza-se o aumento do volume dentro de um compartimento (causado por fraturas, lesão vascular e de tecidos moles), bem como a redução desse volume (queimaduras e reparação de hérnias em pontos frágeis) ${ }^{5}$. No caso relatado, a paciente vítima de queimadura apresentava redução do volume compartimental, estando incluída na incidência anual dessa síndrome aguda em extremidade traumatizada (7,3 por 100.000 homens e de 7,0 por 100.000 mulheres) $)^{6}$. Relativamente ao número de complicações como síndrome compartimental, amputações, rabdomiólise e insuficiência renal aguda, a existência dessas foi frequente entre as vítimas de trauma elétrico, com uma taxa de $72,3 \%$ de técnicas de desbridamento e $58,2 \%$ de enxertos de pele realizados. Acrescenta-se que, semelhante à paciente do caso relatado (29 anos), a idade média das vítimas foi de 30,4 anos, e a taxa de mortalidade foi de $8,2 \%{ }^{7}$.

Por conseguinte, diante de um paciente com contexto sintomatológico semelhante ao estudado, o primeiro passo consiste em reconhecer a síndrome, realizar o diagnóstico precocemente e manejar a doença de forma prática e resolutiva. A partir disso, como tratamento mais apropriado, a fasciotomia descompressiva 
apresenta bons resultados, pois atua contra o principal fator desencadeante da síndrome, ou seja, o aumento da pressão compartimental ${ }^{2}$. Também é essencial aplicar todas as condutas de precaução, a exemplo da implementação de antibioticoterapia adequada e exploração de toda a região compartimental para verificação da presença da síndrome em todo o segmento lesionado ${ }^{7}$. As medidas a serem tomadas devem ser compreendidas por toda a equipe multiprofissional de saúde, visto que a evolução patológica desse quadro clínico pode levar a desfechos mais graves e até à morte.

O presente trabalho serve de alerta a todos os médicos - especialmente cirurgiões, por lidarem constantemente com essa patologia - para a importância de reconhecer a presença da síndrome em regiões não comumente acometidas. Desse modo, o profissional médico deve estar atento aos sinais apresentados pelo paciente, bem como ao manejo correto e eficaz dessa doença, visando à resolução desse quadro patológico.

\section{CONCLUSÃO}

O presente trabalho ilustra a conduta referente a uma paciente feminina, de 29 anos, inicialmente admitida com quadro álgico de forte intensidade em mão esquerda após queimadura com café quente. O exame físico demonstrou lesão ao nível da região anterior à tabaqueira anatômica de tamanho significativo com crosta áspera e fragilidade funcional dos dedos da mão acometida. Por se tratar de um quadro grave de síndrome compartimental, foi realizado o desbridamento da região, porém houve retorno da paciente com piora da dor, sendo realizada imediata exploração profunda da ferida e abertura da fáscia dorsal da mão, corroborando a eficiência do reparo operatório.

\section{REFERÊNCIAS}

1. Oliveira RA, Nigri EL. Sutura elástica no tratamento de escarotomias e fasciotomias de pacientes queimados. Rev Bras Queimaduras. 2012;11(2):63-6.
2. Baumfeld D, Pereira A, Lage C, Miura G, Gomes Y, Nery C. Síndrome compartimental aguda não traumática em atleta de futebol tratada por fasciotomia descompressiva segmentar: relato de caso. Rev Bras Ortop. 2018;53(2):244-7. http://dx.doi.org/10.1016/j. rbo.2017.02.005. PMid:29911093.

3. Maciel A, Dominguete $M$, Silva J No. Anestésico tópico para liberação de pontos-gatilhos em síndrome dolorosa miofascial. BrJP. 2018;1(2):147-50

4. Via AG, Oliva F, Spoliti M, Maffulli N. Acute compartment syndrome. Muscles Ligaments Tendons J. 2015;5(1):18-22. PMid:25878982.

5. Carvalho CM, Faria GEL, Milcheski DA, Gomez DS, Ferreira MC. Estudo clínico epidemiológico de vítimas de queimaduras elétricas nos últimos 10 anos. Rev Bras Queimaduras. 2012;11(4):230-3.

6. Henríquez J, Yáñez G, Searle S. Alternativas de cierre de fasciotomías en extremidades. Rev Chil Cir. 2018;70(2):185-93. http://dx.doi. org/10.4067/s0718-40262018000200185.

7. Mendes R, Pedro I, Sousa A. Síndrome compartimental do antebraço. Rev Port Ortop Traumatol. 2014;22(1):127-34.

Correspondência Clara Wilma Fernandes Rosendo Universidade Federal do Rio Grande do Norte - UFRN, Departamento de Ciências da Saúde Rua Doutor Carlos Passos, 1746 - Tirol CEP 59015-310 - Natal (RN), Brasil Tel.: (84) 98734-1544 E-mail: clararondes@hotmail.com

Informações sobre os autores CWFR - Acadêmica de Medicina, Departamento de Ciências da Saúde, Universidade Federal do Rio Grande do Norte (UFRN). JRLPC - Docente Adjunto IV, Curso de Medicina, Universidade do Estado do Rio Grande do Norte (UERN).

RRO - Mestre em Patologia, Universidade Federal de Pernambuco (UFPE); Cirurgião Vascular, Departamento de Cirurgia Geral e Vascular, Hospital Regional Tarcísio Maia (HRTM).

Contribuições dos autores Concepção e desenho do estudo: CWFR Análise e interpretação dos dados: RRO Coleta de dados: RRO Redação do artigo: CWFR Revisão crítica do texto: JRLPC Aprovação final do artigo*: RRO Análise estatística: JRLPC Responsabilidade geral pelo estudo: RRO

*Todos os autores leram e aprovaram a versão final submetida ao J Vasc Bras. 